\title{
Adaptive CSLBP compressed image hashing
}

\author{
Varsha Patil, Tanuja Sarode \\ Department of Computer Engineering, TSEC, Mumbai University, India
}

\begin{tabular}{|c|c|}
\hline Article Info & ABSTRACT \\
\hline & \multirow{10}{*}{$\begin{array}{l}\text { Hashing is popular technique of image authentication to identify malicious } \\
\text { attacks and it also allows appearance changes in an image in controlled way. } \\
\text { Image hashing is quality summarization of images. Quality summarization } \\
\text { implies extraction and representation of powerful low level features in } \\
\text { compact form. Proposed adaptive CSLBP compressed hashing method uses } \\
\text { modified CSLBP (Center Symmetric Local Binary Pattern) as a basic method } \\
\text { for texture extraction and color weight factor derived from L*a*b* color } \\
\text { space. Image hash is generated from image texture. Color weight factors are } \\
\text { used adaptively in average and difference forms to enhance discrimination } \\
\text { capability of hash. For smooth region, averaging of colours used while for } \\
\text { non-smooth region, color differencing is used. Adaptive CSLBP histogram is } \\
\text { a compressed form of CSLBP and its quality is improved by adaptive color } \\
\text { weight factor. Experimental results are demonstrated with two benchmarks, } \\
\text { normalized hamming distance and ROC characteristics. Proposed method } \\
\text { successfully differentiate between content change and content persevering } \\
\text { modifications for color images. }\end{array}$} \\
\hline Received Nov 25, 2018 & \\
\hline Revised Mar 28, 2019 & \\
\hline Accepted Apr 3, 2019 & \\
\hline Keywords: & \\
\hline Authentication & \\
\hline CSLBP & \\
\hline Histogram & \\
\hline Image hashing & \\
\hline $\mathrm{L} * \mathrm{a} * \mathrm{~b} *$ color model & \\
\hline
\end{tabular}

Copyright @ 2019 Institute of Advanced Engineering and Science. All rights reserved.

Corresponding Author:

Varsha Patil,

Department of Computer Engineering,

TSEC, Mumbai University, India.

Email: varshasp2977@gmail.com

\section{INTRODUCTION}

Success and popularity of digital technology is enormous. Digital forgery (tampering) and unauthorized use have reached a significant level that makes multimedia authentication and security very challenging and demanding. Some of this data is confidential and there is need of protecting and verifying the data integrity. It is necessary to protect some data for its confidentiality and integrity. In cryptography, hashing techniques are there for data integrity. These methods are basically designed for text data and follow stringent approach in which even change in single bit drastically causes change in its hash code. Such techniques cannot be utilized for digital data like image, video etc. as limited change is common on these data types. Limited change in the image data indicates content preserving operations like gamma correction, scaling, contrast modification etc. To deal with data integrity issues, image hashing is simple and efficient solution. Content change in an image is treated as malicious operation. The hash code of original and modified image is drastically different or above prescribed threshold when some malicious changes occur in an image [1-4].

Most of the existing image hashing methods target only gray scale images. The proposed hashing method is designed for colour images. For colour image hashing, color is an important feature. However, relying only on colour feature for feature extraction is not sufficient. Texture is a very useful depiction for a wide range of images. Colour is highly correlated, specially RGB colour model whereas structures are uncorrelated and random in nature.

Proposed method extracts spatial texture features using modified CSLBP which mainly concentrates on pixel statistics to determine texture strength and pattern. Colour features are fastened in modified CSLBP 
texture descriptor. Colour features are adaptively used based on local region analysis. Color feature is pixel dependent while texture features are determined from set or pixels or neighbourhood. CIE L*a*b* color space is selected for color features as this color model satisfy the perceptual uniformity property. To determine smoothness of local region, Canny edge detector is used. For smooth region, colour averaging is used which represents mean of neighbourhood. For edge dominant region, color differencing is used which represents gradient of neighbourhood. Luminance (L) channel of the lab color space is essentially the gray scale of original RGB image. Texture features is extracted using modified CSLBP on gray scale image (Luminance channel).

As number of features increases, hashing algorithm becomes more robust and gives desirable discrimination quality. However, with increase in features, size of hash also increases which is not acceptable. To overcome this problem, color and texture features are not used separately but color feature is superimposed on texture feature.

Various researchers studied image hashing in terms of quality feature extraction and their compact representation. Pairing local and global features together is quite robust and popular approach for image hashing as it identifies content change at local as well as at global level. Following represents various global and local features pairs for content change location locally as well as globally. DWT-SVD and Saliency object detection using spectral residual model; Projected Gradient Non-negative Matrix Factorization (PGNMF), ring partition and saliency detection; Zernike moment and Salient point detection; Zernike moment and Haralick local features; Zernike moments, MOD-LBP and Haralick texture features; Invariant moments from Radon coefficients and statistical measures from Radon coefficients; DCT coefficients of Watson's visual model and SIFT key points; Color vector angle and Salient edge points [5-12].

Transform is an very efficient way to separate out components from an image. These components are sensitive to content change and robust to content preserving. These components can be easily represented in hash form by applying simple operations. Fourier-Mellin transformed (FMT) image is converted into polar co-ordinates. From polar co-ordinates, features are extracted and quantized to generate a binary hash [13]. To improve the imperceptibility aspect in cryptography, combination of DCT and DWT transformed is used and double protection on the digital message is achieved by OTP encryption [14]. To provide protection from attacks, wavelet based Least Significant Bit Watermarking (WLSBWM) integrates the alphabet pattern approach which generated the shuffled image and wavelet concept to reduce the dimensionality of watermark [15]. Texture feature is extracted from Wave atom transform having characteristics of sparser expansion. Gray code optimization and chaotic map quantization is performed [16]. Sub band images are generated by applying 2-level DWT on the input color image. $\mathrm{LL}_{2}$ sub-band image arranged in concentric rings to extract features for hash creation [17]. Features such as Discrete Cosine Transformation (DCT) and Gray Level Co-occurrence Matrix (GLCM) are extracted in circular rings to generate rotation invariant hash [18].

For color image hashing approaches color represents important feature to detect changes. Perceptual color difference is captured by color vector angle which is used to generate hash. Secondary image is generated from color vector angle. Mean is calculated from non overlapping blocks of secondary image and further compressed by DWT to generate compact hash [19]. From HSI plane, secondary HSI quantized image is generated. 24 bin histogram is generated from quantized HSI histogram to represent hash. This method considers purely global features and hence, performance is limited for various attacks [20]. From HSI and YCbCr colour space, block mean and variance are obtained. Euclidean distance is calculated between block features and reference features and treated as a image hash [21]. Three histograms are generated for an color image. Histogram captures specific distribution of pixel over the image which is measured as four moment like mean, standard deviation, skewness and kurtosis to generate hash [22].

Local Binary Pattern (LBP) texture descriptor is popular because of its computational simplicity, tolerance for illumination changes, rotation and scale invariance. However it generates histogram of 256 bin which makes inappropriate choice an image hashing [23, 24]. Image hashing using Centre Symmetric Local Binary Pattern (CSLBP) [25] is suitable option as it generates histogram of 16 bin. In CSLBP, to extract texture, only sign difference of four cross symmetric pairs is taken. Davarzani et al. [26] used sign as well as magnitude difference of four cross symmetric pairs. In this approach, authors generated four histogram for each direction with magnitude as weight factor, which resulted in total histogram of 64 bin. The 64 bin histogram violates compact length property of image hash as well as magnitude weight on each histogram did not enhance discrimination capability. CSLBP histogram can be compressed by flipped difference concept [27]. Compression of plain histogram gives poor discrimination results. To get desirable discrimination of hashing, local weight factors are used during histogram correction. In our previous approaches, various types of weight factors are used to enhance discrimination. In AQ-CSLBP [28], instead of separate magnitude, the average of magnitude difference of four cross symmetric pairs is used as weight factor. In SDQ-CSLBP [29], weight factor is standard deviation of four cross symmetric is used. 
Similar for CoCQ- CSLBP [30], correlation coefficient between reference local area and image local area represents weight factor. Finally, in LoGQ-CSLBP [31], Laplacian of Gaussian (LoG) of local area which is robust to noise is used as a weight factor. LBP can easily extended to color images. In Color LBP [32], the operator is used on each color channel independently, and then for pairs of color channels in which center pixel is taken from one channel and the neighbouring pixels from the other channel. Therefore by this method total nine histogram are generated. Size of resultant descriptor is huge. However opposing pairs, such as R-G and G-R are highly redundant, so either of them can be used in the analysis. This result in total six histograms ( $\mathrm{R}$ channel, G channel, B channel, RG channel, RB channel, GB channel). Generated feature vector is six times larger than LBPs. For color images, various combinations of LBP are available like RGB-LBP, nRGB-LBP, Transformed color LBP, Opponent LBP, nOpponent-LBP, Hue-LBP [33]. In Improved Opponent Colour [34], intra and inter channel features are considered. In this method, thresholding is done against the average value. In experimental result analysis section, we showed that, all these color LBP variant approaches are not suitable for image hashing due its long length and poor discrimination power.

\section{PROPOSED METHOD}

In the proposed approach, the input RGB color image is converted to $\mathrm{L}^{*} \mathrm{a} * \mathrm{~b} *$ color space for color features extraction. Luminance channel of Lab color space is used by CSLBP and Canny edge detector. CSLBP extracts texture features and Canny edge detector detects presence of gradient information in local region.

\subsection{Pre-processing}

Initially, the input RGB color image is converted to a fixed size by using bilinear interpolation. Image resizing is necessary for experimental analysis and comparison with other methods. Also it is necessary to ensure that, images with different resolutions will have similar hash code. To enhance robustness against content preserving manipulations, input image is filtered by Gaussian filter. A $3 \times 3$ Gaussian filter mask is convolved over the entire image. By doing convolution operation, it reduces disturbance caused by manipulations like noise, lossy compression.

\subsection{Modified CSLBP}

CSLBP considers only cross symmetric pairs which captures rotation invariant texture details and also produces histogram with less no. of bin. CSLBP considers signed gray level di-erences of cross symmetric pixel pairs multiplied by powers of two in a particular direction. For $3 \times 3$ local area, CSLBP value for a pixel lies in the range from 0 to 15 , which leads to 16 bin histogram at semi global level. CSLBP is suitable choice for hashing for number of reasons. First it generates small histogram, captures improved texture information, provides robustness on flat areas. Equations (1) and (2) represents CSLBP.

$$
\begin{aligned}
& \operatorname{CSLBP}_{P, R, T}\left(g_{c}\right)=\sum_{p=0}^{P / 2-1} s\left(g_{p}-g_{(P / 2)}\right) 2^{p} \\
& \operatorname{sign}\left(g_{p}-g_{p+(P / 2)}\right)=\left\{\begin{array}{lc}
1, & \left(g_{p}-g_{p+(P / 2)}\right)>T \\
O, & \text { otherwise }
\end{array}\right.
\end{aligned}
$$

where, T: threshold; R: radius; $\mathrm{P}$ : no. of neighbours; $\mathrm{g}_{\mathrm{c}}$ : center pixel; $\mathrm{g}_{\mathrm{p}}$ : neighbours of centre pixel; $\mathrm{s}\left(\mathrm{g}_{\mathrm{p}}-\mathrm{g}_{\mathrm{p}+(\mathrm{P} / 2)}\right)$ : sign function of CSLBP; CSLBP: CSLBP texture extractor. Values of parameters are set as $\mathrm{T}=0.1, \mathrm{P}=8, \mathrm{R}=1$.

In modified CSLBP, we divided eight neighbours into two types, four immediate neighbours and four diagonal neighbours. Immediate neighbours are at distance of 1 unit from centre pixel while diagonal neighbours are at distance of $(\sqrt{2})$ unit. Signed differences of cross symmetric pairs is taken for four immediate neighbours and for four diagonal neighbours separately as shown in Equations (3) and (4).

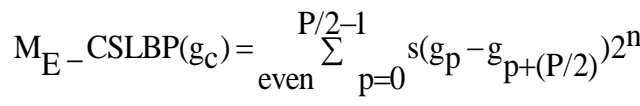

$$
\begin{aligned}
& \mathrm{M}_{\mathrm{O}-} \operatorname{CSLBP}\left(\mathrm{g}_{\mathrm{c}}\right)={ }_{\text {odd }}^{\mathrm{P} / 2-1} \sum_{\mathrm{p}=1}^{\mathrm{s}} \mathrm{s}\left(\mathrm{g}_{\mathrm{p}}-\mathrm{g}_{\mathrm{p}+(\mathrm{P} / 2)}\right) 2^{\mathrm{n}}
\end{aligned}
$$


where $\mathrm{M}_{\mathrm{E}}-\mathrm{CSLBP}$ is CSLBP for nearest neighbours; $\mathrm{M}_{\mathrm{O}}-\mathrm{CSLBP}$ is CSLBP for diagonal neighbours; $\mathrm{n}$ is unit increment operator; $p$ is even increment operator for nearest neighbours and odd increment operator for diagonal neighbours; $\mathrm{P}$ represents neighbours of center pixel; $\mathrm{s}\left(\mathrm{g}_{\mathrm{p}}-\mathrm{g}_{\mathrm{p}+(\mathrm{P} / 2)}\right)$ is sign function

In this proposed approach, like CSLBP, histogram is constructed by taking signed differences of cross symmetric pairs. But unlike CSLBP, it generates 8 bin histogram without any quality reduction. This gives $50 \%$ reduction in hash size with same discrimination capability that of 16 bin CSLBP. After MCSLBP calculation for all pixels in an image. Histogram is constructed at semi global level. For every block, two histogram generated of four bin, one for immediate and other for diagonal neighbours. To further enhance discrimination power, adaptive color weight factors from Lab color space is used.

\subsection{Color weight factor}

Color is the most dominant and distinguishing visual feature. Drawback of the RGB color space is high correlation between planes. $\mathrm{L} * \mathrm{a} * \mathrm{~b} *$ or Lab color space is a color-opponent space with dimensions $\mathrm{L}$ for lightness and $\mathrm{a}$ and $\mathrm{b}$ for the color-opponent dimensions. Lab color space satisfies perceptual uniformity property at local level. A perceptual uniform color space ensures that the difference between two colors (as perceived by the human eye) is proportional to the Euclidian distance within the given color space. As color weight factors are selected at local level, total advantage of perceptual uniformity property is utilized.

For local region $3 \times 3$, Canny edge detector is applied to find edge details. Adaptive averaging and difference weight factor is selected based on response of canny edge detectors for four neighbours. For smooth region, color averaging weight factor is used while for non-smooth region color differencing weight factor is used.

- For 4 nearest neighbours

$$
\text { Canny Edge Output }=\left[\mathrm{CE}_{0} ; \mathrm{CE}_{4} ; \mathrm{CE}_{2} ; \mathrm{CE}_{6}\right]
$$

where CE represent Canny edge output either one or zero which shows region is either smooth or presence of edge points. $\mathrm{CE}_{0}, \mathrm{CE}_{4}, \mathrm{CE}_{2}$ and $\mathrm{CE}_{6}$ represents nearest neighbours around a center pixel. For local region of $3 \times 3$ of LAB color image, neighbourhood is represented as below

Odd neighbours: [ $\left.\mathrm{L}_{0}, \mathrm{~A}_{0}, \mathrm{~B}_{0}\right],\left[\mathrm{L}_{2}, \mathrm{~A}_{2}, \mathrm{~B}_{2}\right],\left[\mathrm{L}_{4}, \mathrm{~A}_{4}, \mathrm{~B}_{4}\right],\left[\mathrm{L}_{6}, \mathrm{~A}_{6}, \mathrm{~B}_{6}\right]$

Even neighbours: $\left[\mathrm{L}_{1}, \mathrm{~A}_{1}, \mathrm{~B}_{1}\right],\left[\mathrm{L}_{3}, \mathrm{~A}_{3}, \mathrm{~B}_{3}\right],\left[\mathrm{L}_{5}, \mathrm{~A}_{5}, \mathrm{~B}_{5}\right],\left[\mathrm{L}_{7}, \mathrm{~A}_{7}, \mathrm{~B}_{7}\right]$

- For smooth region

$$
\begin{aligned}
& \mathrm{AVG}_{\text {nearest }}=\sqrt{\left(\mathrm{L}_{\mathrm{avg}}+\mathrm{A}_{\mathrm{avg}}+\mathrm{B}_{\mathrm{avg}}\right)} \\
& \mathrm{L}_{\mathrm{avg}}=\left(\mathrm{L}_{0}^{2}+\mathrm{L}_{2}^{2}+\mathrm{L}_{4}^{2}+\mathrm{L}_{6}^{2}\right) / 4 \\
& \mathrm{~A}_{\mathrm{avg}}=\left(\mathrm{A}_{0}^{2}+\mathrm{A}_{2}^{2}+\mathrm{A}_{4}^{2}+\mathrm{A}_{6}^{2}\right) / 4 \\
& \mathrm{~B}_{\mathrm{avg}}=\left(\mathrm{B}_{0}^{2}+\mathrm{B}_{2}^{2}+\mathrm{B}_{4}^{2}+\mathrm{B}_{6}^{2}\right) / 4
\end{aligned}
$$

- For non-smooth region

$$
\begin{aligned}
& \text { Diff }_{\text {nearest }}=\sqrt{\left(\mathrm{L}_{\text {diff }}+\mathrm{A}_{\text {diff }}+\mathrm{B}_{\text {diff }}\right)} \\
& \mathrm{L}_{\text {diff }}=\left(\mathrm{L}_{0}-\mathrm{L}_{4}\right)\left(\mathrm{L}_{0}-\mathrm{L}_{4}\right)+\left(\mathrm{L}_{2}-\mathrm{L}_{6}\right)\left(\mathrm{L}_{2}-\mathrm{L}_{6}\right) \\
& \mathrm{A}_{\text {diff }}=\left(\mathrm{A}_{0}-\mathrm{A}_{4}\right)\left(\mathrm{A}_{0}-\mathrm{A}_{4}\right)+\left(\mathrm{A}_{2}-\mathrm{A}_{6}\right)\left(\mathrm{A}_{2}-\mathrm{A}_{6}\right) \\
& \mathrm{B}_{\text {diff }}=\left(\mathrm{B}_{0}-\mathrm{B}_{4}\right)\left(\mathrm{B}_{0}-\mathrm{B}_{4}\right)+\left(\mathrm{B}_{2}-\mathrm{B}_{6}\right)\left(\mathrm{B}_{2}-\mathrm{B}_{6}\right)
\end{aligned}
$$


where $\mathrm{L}_{\mathrm{avg}}, \mathrm{A}_{\mathrm{avg}}, \mathrm{B}_{\mathrm{avg}}$ are averages of even components of $\mathrm{L}, \mathrm{A}$ and $\mathrm{B}$ color space respectively; Avg nearest is summation of $\mathrm{L}_{\mathrm{avg}}, \mathrm{A}_{\mathrm{avg}}, \mathrm{B}_{\mathrm{avg}}$; $\mathrm{L}_{\text {diff, }} \mathrm{A}_{\text {diff, }}, \mathrm{B}_{\text {diff }}$ are squared difference of cross symmetric pairs of even components of $\mathrm{L}, \mathrm{A}$ and $\mathrm{B}$ color space respectively; Diff ${ }_{\text {nearest }}$ is summation of $\mathrm{L}_{\text {diff }}, \mathrm{A}_{\text {diff }}, \mathrm{B}_{\text {diff }}$

- For 4 diagonal neighbours

$$
\text { Canny Edge Output }=\left[\mathrm{CE}_{1} ; \mathrm{CE}_{3} ; \mathrm{CE}_{5} ; \mathrm{CE}_{7}\right]
$$

where CE represent Canny edge output either one or zero which shows region is either smooth or presence of edge points. $\mathrm{CE}_{1}, \mathrm{CE}_{3}, \mathrm{CE}_{5}$ and $\mathrm{CE}_{7}$ represents diagonal neighbours around a center pixel.

- For smooth region

$$
\begin{aligned}
& \mathrm{AVG}_{\text {diagonal }}=\sqrt{\left(\mathrm{L}_{\mathrm{avg}}+\mathrm{A}_{\mathrm{avg}}+\mathrm{B}_{\mathrm{avg}}\right)} \\
& \mathrm{L}_{\mathrm{avg}}=\left(\mathrm{L}_{1}^{2}+\mathrm{L}_{3}^{2}+\mathrm{L}_{5}^{2}+\mathrm{L}_{7}^{2}\right) / 4 \\
& \mathrm{~A}_{\mathrm{avg}}=\left(\mathrm{A}_{1}^{2}+\mathrm{A}_{3}^{2}+\mathrm{A}_{5}^{2}+\mathrm{A}_{7}^{2}\right) / 4 \\
& \mathrm{~B}_{\mathrm{avg}}=\left(\mathrm{B}_{1}{ }_{1}+\mathrm{B}_{3}^{2}+\mathrm{B}_{5}^{2}+\mathrm{B}^{2}\right) / 4
\end{aligned}
$$

- For non-smooth region

$$
\begin{aligned}
& \text { Diff }_{\text {diagonal }}=\sqrt{\left(\mathrm{L}_{\text {diff }}+\mathrm{A}_{\text {diff }}+\mathrm{B}_{\text {diff }}\right)} \\
& \mathrm{L}_{\text {diff }}=\left(\mathrm{L}_{1}-\mathrm{L}_{5}\right)\left(\mathrm{L}_{1}-\mathrm{L}_{5}\right)+\left(\mathrm{L}_{3}-\mathrm{L}_{7}\right)\left(\mathrm{L}_{3}-\mathrm{L}_{7}\right) \\
& \mathrm{A}_{\text {diff }}=\left(\mathrm{A}_{1}-\mathrm{A}_{5}\right)\left(\mathrm{A}_{1}-\mathrm{A}_{5}\right)+\left(\mathrm{A}_{3}-\mathrm{A}_{7}\right)\left(\mathrm{A}_{3}-\mathrm{A}_{7}\right) \\
& \mathrm{B}_{\text {diff }}=\left(\mathrm{B}_{1}-\mathrm{B}_{5}\right)\left(\mathrm{B}_{1}-\mathrm{B}_{5}\right)+\left(\mathrm{B}_{3}-\mathrm{B}_{7}\right)\left(\mathrm{B}_{3}-\mathrm{B}_{7}\right)
\end{aligned}
$$

where $\mathrm{L}_{\mathrm{avg}}, \mathrm{A}_{\mathrm{avg}}, \mathrm{B}_{\mathrm{avg}}$ are averages of odd components of $\mathrm{L}, \mathrm{A}$ and $\mathrm{B}$ color space respectively; Avg nearest is summation of $\mathrm{L}_{\mathrm{avg}}, \mathrm{A}_{\text {avg }}, \mathrm{B}_{\text {avg }}$; $\mathrm{L}_{\text {diff, }} \mathrm{A}_{\text {diff, }}, \mathrm{B}_{\text {diff }}$ are squared difference of cross symmetric pairs of odd

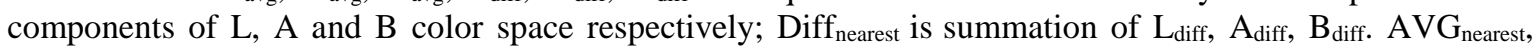
$\mathrm{AVG}_{\text {diagonal, }} \mathrm{DIFF}_{\text {nearest }}$ and $\mathrm{DIFF}_{\text {diagonal }}$ are converted into weight as given in Table 1.

Table 1. Delta E values and their weight

\begin{tabular}{ccc}
\hline Value & Meaning & Weight \\
\hline $0-1$ & A normally invisible difference & 10 \\
$1-2$ & Very small difference, only obvious to a trained eye & 20 \\
$2-3.5$ & Medium difference, also obvious to an untrained eye & 30 \\
$3.5-5$ & An obvious difference & 40 \\
$>6$ & A very obvious difference & 50 \\
\hline
\end{tabular}

$$
\begin{aligned}
& \mathrm{A}=\mathrm{AVG}_{\text {immediate }}+\mathrm{AVG}_{\text {diagonal }} \\
& \mathrm{D}=\mathrm{DIFF}_{\text {immediate }}+\mathrm{DIFF}_{\text {diagonal }}
\end{aligned}
$$

where $\mathrm{A}$ and $\mathrm{D}$ represents color weigh factors which acts as boosting agent during $\mathrm{M}_{\mathrm{E}-\mathrm{CSLBP}}$ and $\mathrm{M}_{\mathrm{O}-\mathrm{CSLBP}}$ histogram construction for sub-block size $\mathrm{B} \times \mathrm{B}$ respectively. Final equation of $\mathrm{M}_{\mathrm{E}}-\mathrm{CSLBP}$ and $\mathrm{M}_{\mathrm{O}}-\mathrm{CSLBP}$ histogram are as below. 


$$
\begin{aligned}
& \mathrm{H}_{\mathrm{E}-\operatorname{CSLBP}}(\mathrm{b})=\sum_{i=1}^{B} \sum_{j=1}^{B} A(i, j) \times f\left(M_{E-\operatorname{CSLBP}}(i, j), b\right) \\
& \mathrm{H}_{\mathrm{O}-\operatorname{CSLBP}}(\mathrm{b})=\sum_{i=1}^{B} \sum_{j=1}^{B} D(i, j) \times f\left(M_{O-\operatorname{CSLBP}}(i, j), b\right)
\end{aligned}
$$

where $B$ is size of the sub-block set to $32 \times 32$ and value of $b$ varies from 0 to 3 .

For each sub-block two histograms are constructed. Each histogram is quantized to generate binary output. To generate final image binary hash, binary output of all histograms is concatenated.

\section{EXPERIMENTAL RESULTS AND ANALYSIS}

In this section, experimental results for the proposed method and its comparative methods are presented. Sensitivity to content change and robustness to content preserving are checked with two benchmarks, first is Normalized Hamming Distance (NHD) and other is Receiver operating characteristics (ROC). NHD shows how much hash code of original and forged images are vary for malicious and non malicious operations. However if normalized hamming distance is similar for both types of operations i.e. malicious and non-malicious attacks, it indicates poor performance of the algorithm. Second benchmark is ROC which indicates the discrimination power of hashing algorithm. TPR (True Positive Rate) should be high for less FPR (False Positive Rate) for desirable discrimination. The original database contains 23 color images taken from internet and Matlab standard directory. Image size taken as $256 \times 256$ for analysis purpose with other methods. Variety types of attacks are applied on original image database to generate new database which contain malicious as well as non malicious images based on intensity of attacks. Total 61 operations are applied to generate $23 \times 61=1403$ images in which some are authentic images as content is preserved and some are non-authentic as content is changed. Various attacks and their parameters are given in following given in following Table 2. Table 2 also indicates symbolic names for various attacks for showing results in simplified manner. Table 3 and Table 4 shows NHD and ROC observations for various attacks mentioned in Table 2 for the proposed method.

Table 2. Various attacks, parameter, and their symbolic names

\begin{tabular}{lll}
\hline \multicolumn{1}{c}{ Operations } & \multicolumn{1}{c}{ Descriptions } & \multicolumn{1}{c}{ Parameters } \\
\hline Cropping (A) & Ratio & $1 \%, 3 \%, 5 \%, 7 \%, 9 \%$ \\
Salt \& Pepper Noise (B) & Noise Density & $0.01,0.02,0.03,0.05,0.1$ \\
Gaussian Noise (C) & Noise Variance & $0.001,0.005,0.01,0.02,0.05$ \\
Scaling (D) & Scaling factor & $0.7,0.8,0.9,1.1,1.2,0.01,0.05,0.10,0.15,0.20$ \\
Rotate (E) & Rotation Angle & $2^{0}, 4^{0}, 6^{0}, 8^{0}, 10^{0}$ \\
JPEG Compression (F) & Quality Factor & $10,30,50,70,90$ \\
Gamma Correction (G) & Gamma value & $0.75,0.8,0.9,1.1,1.25,4.25,4.50,4.75,5.00,5.25$ \\
Increase Brightness (H) & Range of adjustment & {$[0.81],[0.61],[0.41],[0.21]$} \\
Decrease Brightness (I) & Range of adjustment & {$[0.6],[00.4],[0.2],[0.1]$} \\
Increase Contrast (J) & Range of adjustment & {$[0.8],[0.6],[00.4],[0.2]$} \\
Decrease Contrast (K) & Range of adjustment & {$[0.81],[0.61],[0.41],[0.21]$} \\
\hline
\end{tabular}

Table 3. NHD for adaptive CSLBP compressed image hashing

\begin{tabular}{lcc}
\hline \multicolumn{1}{c}{ Attack } & $\begin{array}{r}\text { Adaptive CSLBP Compressed Color Image Hashing } \mathrm{T}_{\mathrm{NHD}}=0.10 \\
\text { Auth }\end{array}$ & $\begin{array}{c}\text { Non Auth } \\
\text { Cropping }\end{array}$ \\
Salt \& Pepper Noise & 0.10 & 0.21 \\
Gaussian Noise & 0.07 & 0.17 \\
Scaling & 0.10 & 0.21 \\
Rotate & 0.05 & 0.24 \\
JPEG Compression & 0.16 & 0.24 \\
Gamma Correction & 0.05 & 0.15 \\
Increase Brightness & 0.04 & 0.18 \\
Decrease Brightness & 0.07 & 0.22 \\
Increase Contrast & 0.04 & 0.11 \\
Decrease Contrast & 0.09 & 0.23 \\
\hline
\end{tabular}


Observations: Table 3 results clearly shows that proposed method distinguished between content preserving and content change for $\mathrm{T}_{\mathrm{NHD}}=0.10$. Only for rotation authentic images results are not satisfactory. Also there is sufficient gap between minimum and maximum $\mathrm{T}_{\mathrm{NHD}}$ distance. Minimum is 0.04 and maximum is 0.24 . This difference is also indicates proposed method has distinguish power of separating authentic and non-authentic images.

Table 4. TPR and FPR for adaptive CSLBP compressed color image hashing

\begin{tabular}{ccc}
\hline Attack & TPR & FPR \\
\hline Cropping & 0.65 & 0.04 \\
Salt \& Pepper Noise & 0.93 & 0.19 \\
Gaussian Noise & 0.67 & 0.00 \\
Scaling & 0.98 & 0.09 \\
Rotate & 0.26 & 0.07 \\
JPEG Compression & 1.00 & 0.26 \\
Gamma Correction & 1.00 & 0.11 \\
Increase Brightness & 0.78 & 0.04 \\
Decrease Brightness & 0.97 & 0.26 \\
Increase Contrast & 0.96 & 0.25 \\
Decrease Contrast & 0.91 & 0.13 \\
Avg. Database & 0.87 & 0.11 \\
\hline
\end{tabular}

Observations: Table 4 shows results for average database is $87 \%$. Figure 2 shows that proposed method gives satisfactory results for almost all types of attacks.

In the following section, proposed method results are compared with other methods. Various types of color LBP's are available which is considered here for comparative analysis. These methods are Color LBP [32], variant of LBP's for color images like RGB-LBP, nRGB-LBP, Transformed color LBP, OpponentLBP, nOpponent-LBP, Hue-LBP [33]. All methods based on LBP's have large no. of histogram bin also results shows that color LBP based methods have a very poor discrimination capability. Zhao et al. [20] developed color image hashing based on color histogram generated from HSI quantized image. It purely takes only color global feature. Size of image hash is small but discrimination power is very poor. Zhou et al. [35] developed Spatial-Color Binary Patterns having histogram of 64 bin. Method is designed for background subtraction. It's hash size is small compared to LBP based method however its discrimination capability for authentication application is very poor. Color image hashing methods cannot rely only on color factor, but also combinations of color and other features should be used.

Table 5 clearly shows that for methods number from 1 to 7 , hash size is more and discrimination power spans from very low to average. For method number 8 and 9, histogram bins are less than LBP based method but discrimination power is very low and average respectively. However, for the proposed method 'Adaptive CSLBP Compressed Color Image Hashing', number of histogram bins are only 8 which results in compact hash size of 512 bits. Discrimination power is also desirable for almost all types of attack.

Table 5. Comparison of existing color hashing techniques with the proposed hashing method 'adaptive compressed CSLBP image hashing'

\begin{tabular}{|c|c|c|c|c|c|}
\hline No & Color image hashing methods & Histogram bin & $\begin{array}{l}\text { Image hash size } \\
\text { (bits) }\end{array}$ & $\begin{array}{l}\text { Discrimination } \\
\text { Power (\%TPR) }\end{array}$ & $\begin{array}{l}\text { Symbolic } \\
\text { Name }\end{array}$ \\
\hline 1. & RGB LBP & 768 & 49152 & Low $(58 \%)$ & E1 \\
\hline 2. & nRGB LBP & 768 & 49152 & Very Low $(47 \%)$ & \\
\hline 3. & Transformed Color LBP & 768 & 49152 & Low $(58 \%)$ & \\
\hline 4. & Opponent LBP & 768 & 49152 & Low $(53 \%)$ & E2 \\
\hline 5. & nOpponent LBP & 512 & 32768 & Very Low $(48 \%)$ & \\
\hline 6. & Hue LBP & 256 & 16384 & Very Low $(33 \%)$ & \\
\hline 7. & Color LBP & 1536 & 98304 & Average $(64 \%)$ & E3 \\
\hline 8. & Color Histogram & 24 & 276 & Very Low $(44 \%)$ & E4 \\
\hline 9. & Spatial-Color Binary Pattern & 64 & 4096 & Average $(69 \%)$ & E5 \\
\hline 10. & $\begin{array}{c}\text { Adaptive Compressed CSLBP } \\
\text { Image Hashing }\end{array}$ & 8 & 512 & High $(87 \%)$ & $\mathrm{P} 1$ \\
\hline
\end{tabular}

Methods 1, 4, 7, 8 and 9 are taken into consideration for comparative analysis with the proposed method for various attacks. Symbolic names are given to them as E1, E2, E3, E4 and E5 respectively. For the proposed method symbolic name is P. E stands for existing method while $\mathrm{P}$ stands for proposed method. Following Figure 3 to Figure 14 shows ROC curves for various attacks for existing and proposed methods on ROC benchmark. 


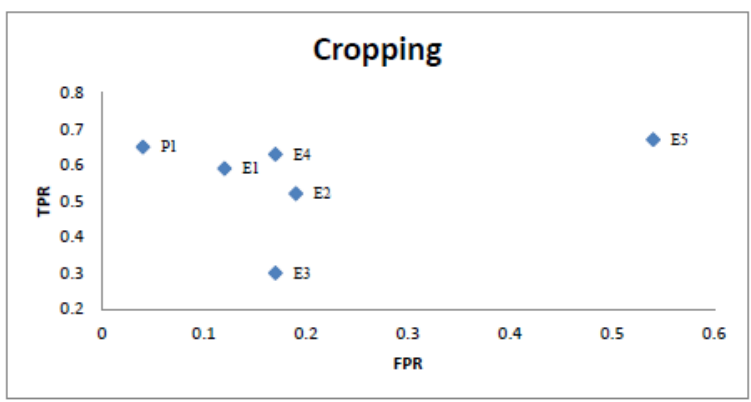

Figure 3. ROC: Cropping

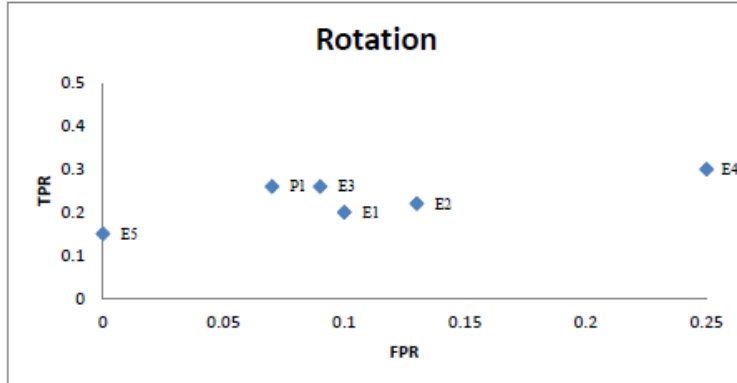

Figure 5. ROC: Gaussian noise

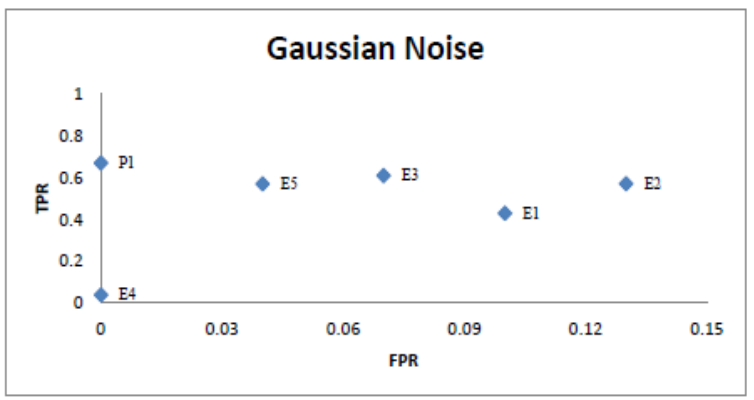

Figure 7. ROC: Rotation

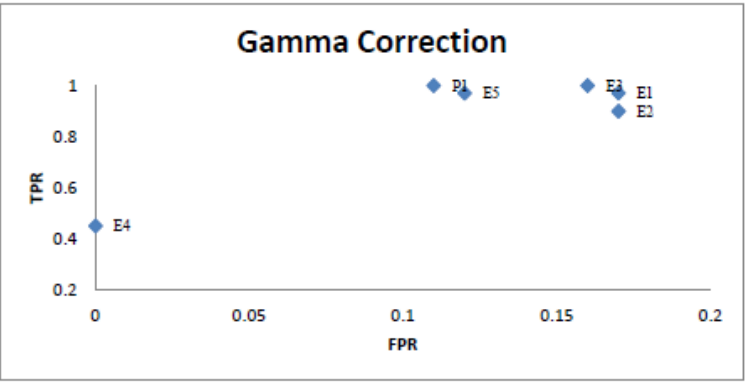

Figure 9. ROC: Gamma correction

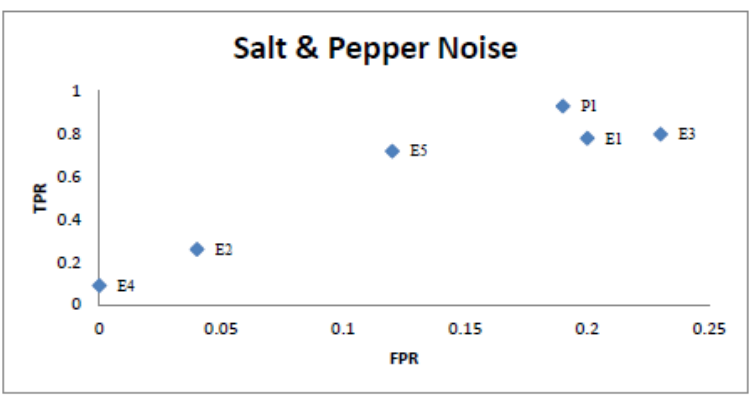

Figure 4. ROC: Salt \& pepper noise

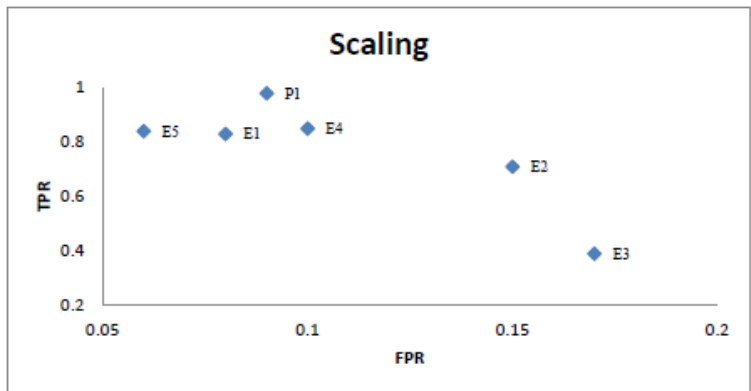

Figure 6. ROC: Scaling

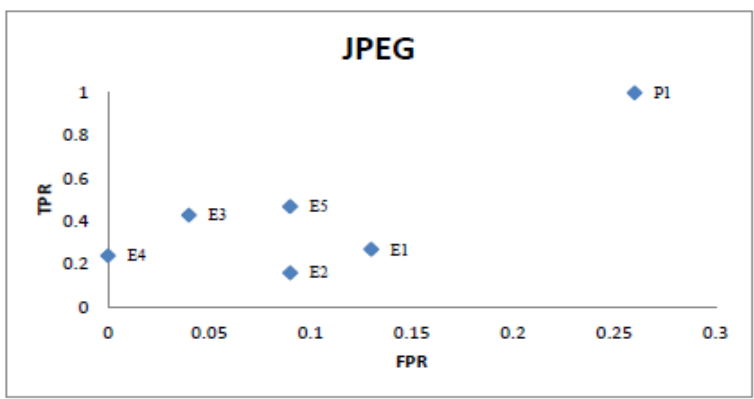

Figure 8. ROC: JPEG

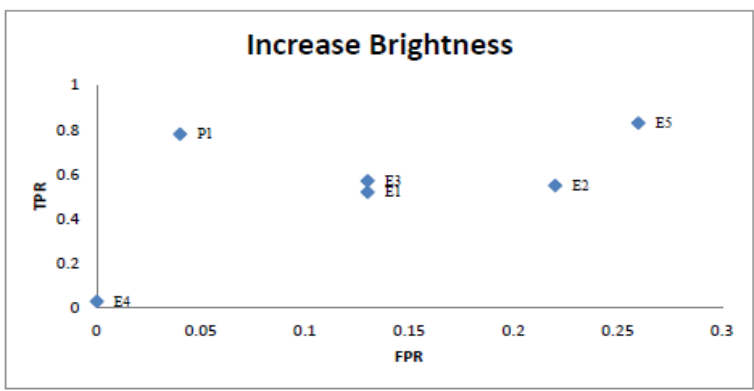

Figure 10. ROC: Increase brightness 


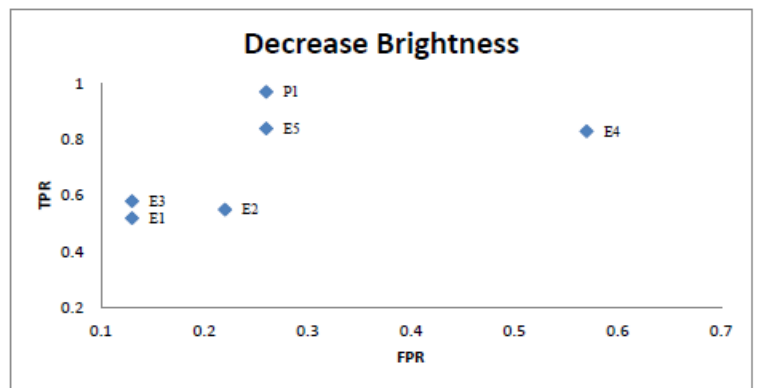

Figure 11. ROC: Decrease brightness

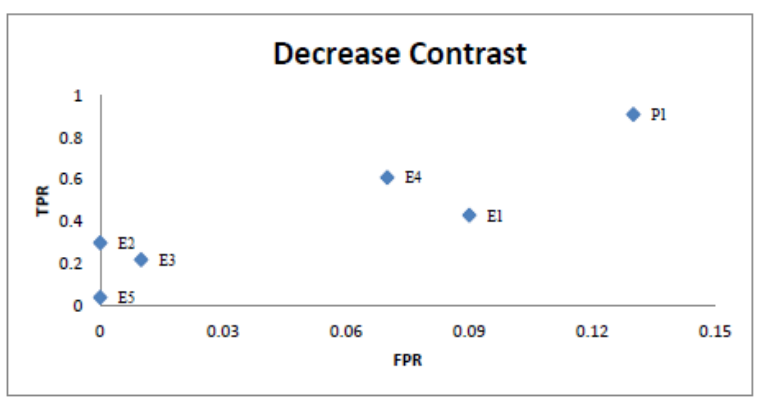

Figure 13. ROC: Decrease contrast

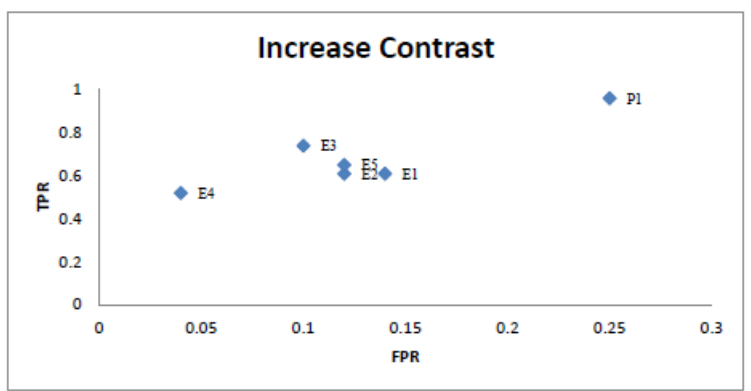

Figure 12. ROC: Increase contrast

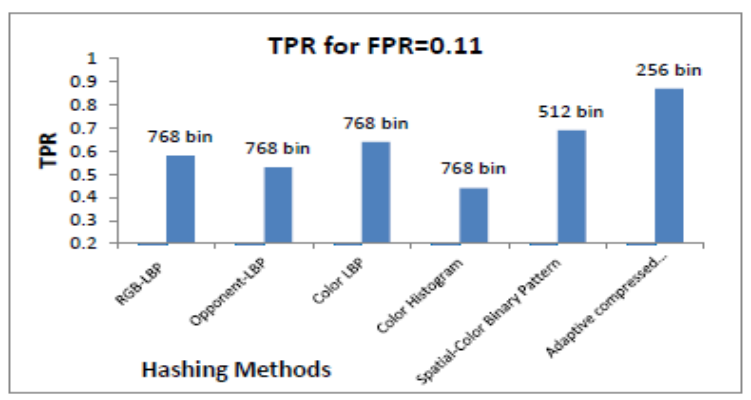

Figure 14. TPR for existing and proposed methods

\section{CONCLUSION}

We have proposed novel image hashing scheme based on combination of texture, color and edge features. As more features are added, hashing scheme becomes more robust at the cost of increased hash size. To achieve compact hash as well as desirable discrimination capability, multiple features are used. In the proposed method, color features are selected adaptively based on the response from edge feature. Color features are not stored separately but super-imposed on texture features as a weight factor. Original CSLBP histogram is compressed by generating two histogram based on location of neighbours and achieved $50 \%$ compression in histogram size without compromise on quality. Results of NHD and ROC shows that proposed method gives satisfactory results for almost all types of attacks.

\section{REFERENCES}

[1] K. B. Adedeji and A. A. Ponnle, "Improved image encryption for real-time application over wireless communication networks using hybrid cryptography technique," Indonesian Journal of Electrical Engineering and Informatics (IJEEI), vol/issue: 4(4), pp. 307-318, 2016.

[2] Sugianto, et al., "Comparison of secret splitting, secret sharing and recursive threshold visual cryptography for security of handwritten images," TELKOMNIKA Telecommunication Computing Electronics and Control, vol/issue: 16(1), pp. 323-333, 2018.

[3] Z. Guojuan and L. Dianji, "An overview of digital watermarking in image forensics," Computational Sciences and Optimization, 2011. CSO 2011. International Conference IEEE, pp. 332-335, 2011.

[4] A. J. Fridrich, et al., "Detection of copy-move forgery in digital images," Digital Forensic Research Workshop, 2003.

[5] R. K. Karsh, et al., "Robust image hashing through DWT-SVD and spectral residual method," EURASIP Journal on Image and Video Processing, vol. 2017, pp. 31, 2017.

[6] R. K. Karsh, et al., "Robust image hashing using ring partition-PGNMF and local features," SpringerPlus Journal, vol. 5, pp. 1995, 2016.

[7] Y. Zhao, et al., "Robust hashing for image authentication using Zernike moments and local features," IEEE Transactions on Information Forensics and Security, vol. 8, pp. 55-63, 2013.

[8] G. Soman, et al., "Block-based forgery detection using global and local features," Soft Computing Systems, 2016. Springer 2016. International Conference Springer, pp. 147-155, 2016.

[9] L. S. Sebastian, et al., "Image authentication by content preserving robust image hashing using local and global features," Elsevier Journal on Procedia Computer Science, vol. 46, pp. 1554-1560, 2015. 
[10] L. I. U. Yuling, et al., "Robust image hashing using radon transform and invariant features," Radioengineering, vol. $25,2016$.

[11] X. Wang, et al., "A visual model-based perceptual image hash for content authentication," IEEE transactions on Information Forensics and Security, vol. 10, pp. 1336-1349, 2015.

[12] C. Qin, et al., "Perceptual hashing for color images based on hybrid extraction of structural features," Elsevier Journal on Signal Processing, vol. 142, pp. 194-205, 2018.

[13] A. Swaminathan, et al., "Robust and secure image hashing," IEEE Transactions on Information Forensics and Security, vol/issue: 1(2), pp. 215-230, 2006.

[14] W. S. Sari, et al., "Comparison of secret splitting, secret sharing and recursive threshold visual cryptography for security of handwritten images," TELKOMNIKA Telecommunication Computing Electronics adn Control, vol/issue: 15(4), pp. 1987-1995, 2017.

[15] V. A. Kumar, et al., "A hybrid digital watermarking approach using wavelets and LSB," International Journal of Electrical and Computer Engineering (IJECE), vol/issue: 7(5), pp. 2483-2495, 2017.

[16] F. Liu, et al., "Wave atom transform generated strong image hashing scheme," Optics Communications, vol/issue: 285(24), pp. 5008-5018, 2012.

[17] L. N. Vadlamudi, et al., "Robust image hashing technique for content authentication based on DWT," Computer Vision and Image Processing, 2017. Internaltional Conferene on Springer, pp. 181-191, 2017.

[18] A. Neelima and K. M. Singh, "A robust image hash function based on color and texture features of the image," Advanced Computing and Communication, 2015, ISACC 2015, International Symposium IEEE, pp. 238-243, 2015.

[19] Z. Tang, et al., "Robust image hashing via colour vector angles and discrete wavelet transform," IET Image Processing, vol. 8, 2014.

[20] Y. Zhao, et al., "Image hashing based on color histogram," Journal of Information \& Computational Science, vol. 9, 2012.

[21] Z. Tang, et al., "Robust image hash function using local color features," EU-International Journal of Electronics and Communications, vol. 67, 2013.

[22] S. Dattatherya, et al., "A generalized image authentication based on statistical moments of color histogram," International Journal on Recent Trends in Engineering \& Technology, vol/issue: 8(1), pp. 40-46, 2013.

[23] T. Ojala, et al., "Multiresolution gray-scale and rotation invariant texture classification with local binary patterns," IEEE transactions on pattern analysis and machine intelligence, vol. 24, 2002.

[24] E. Prasetyo, et al., "Multi-class K-support vector nearest neighbour for mango leaf classification," TELKOMNIKA Telecommunication Computing Electronics adn Control, vol/issue: 16(4), 2018.

[25] J. Xiao and G. Wu, "A robust and compact descriptor based on center-symmetric LBP," Image and Graphics, 2011, ICIG 2011, Sixth International Conference IEEE, pp. 388-393, 2011.

[26] R. Davarzani, et al., "Image authentication using LBP-based perceptual image hashing," Journal of AI and Data Minings, vol. 3, 2015.

[27] J. Baber, et al., "Q-CSLBP: compression of CSLBP descriptor," Multimedia and Information Processing, 2012. Pacific-Rim conference Springer, pp. 513-521, 2012.

[28] V. Patil and T. Sarode, "Image hashing using AQ-CSLBP with double bit quantization," Optoelectronics and Image Processing, 2016. ICOIP 2016. International Conference IEEE, pp. 30-34.

[29] V. Patil and T. Sarode, "Image hashing by SDQ-CSLBP," in Advances in Computing," Communications and Informatics, 2016. ICACCI 2016. International Conference IEEE, pp. 2057-2063, 2016.

[30] V. Patil and T. Sarode, "Image hashing by CCQ-CSLBP," Electrical and Computer Engineering, 2016. WIECONECE 2016. International Conference IEEE, pp. 73-78, 2016.

[31] V. Patil and T. Sarode, "Image hashing by LoG-QCSLBP," Communication and Information Processing, 2016. Second International Conference ACM, pp. 124-128, 2016.

[32] T. Mäenpää and M. Pietikäinen, "Classification with color and texture: jointly or separately?" Pattern Recognition, vol. 37, 2004.

[33] C. Zhu, et al., "Multi-scale color local binary patterns for visual object classes recognition," Pattern Recognition, 2010. ICPR 2010. Twentieth International Conference IEEE, pp. 3065-3068, 2010.

[34] F. Bianconi, et al., "Improved opponent colour local binary patterns for colour texture classification," Computational Color Imaging, 2017, International Workshop Springer, pp. 272-281, 2017.

[35] W. Zhou, et al., "Dynamic background subtraction using spatial-color binary patterns," Image and Graphics, 2011, ICIG 2011. Sixth International Conference IEEE, pp. 314-319, 2011.

\section{BIOGRAPHIES OF AUTHORS}

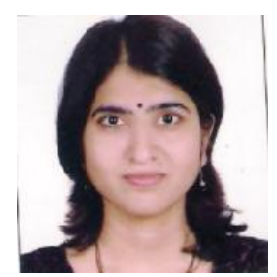

Varsha Patil has received M.E. (Computer Engineering) degree from Mumbai University in 2007, pursuing Ph.D. from Mumbai University, INDIA. She has more than 12 years of experience in teaching. Currently working as Assistant Professor in Computer Engineering Department at South Indian Graduate School of Technology, Mumbai. She is Life member (ISTE). Her areas of interest are Image Processing, Signal Processing and Data Mining, Machine Learning. 
Dr. Tanuja K. Sarode has received M.E. (Computer Engineering) degree from Mumbai University in 2004, Ph.D. from Mukesh Patel School of Technology, Management and Engg. SVKMs NMIMS University, Vile-Parle (W), Mumbai, INDIA in 2010. She has more than 17 years of experience in teaching. Currently working as Professor and Head in Dept. of Computer Engineering at Thadomal Shahani Engineering College, Mumbai. She is Life member (ISTE) and (IETE). Her areas of interest are Image Processing, Signal Processing and Computer Graphics. She has 170 papers in International Conferences/journal to her credit. 